\title{
Search for beta-delayed proton emission from ${ }^{11} \mathrm{Be}$
}

\author{
K. Riisager ${ }^{1 a}$, M.J.G. Borge ${ }^{2,3}$, J.A. Briz ${ }^{3}$, M. Carmona-Gallardo ${ }^{4}$, O. Forstner ${ }^{5}$, L.M. Fraile ${ }^{4}$, H.O.U. Fynbo ${ }^{1}$, A. \\ Garzon Camacho ${ }^{3}$, J.G. Johansen ${ }^{1}$, B. Jonson ${ }^{6}$, M.V. Lund ${ }^{1}$, J. Lachner ${ }^{5}$, M. Madurga ${ }^{2}$, S. Merchel ${ }^{7}$, E. Nacher ${ }^{3}$, T. \\ Nilsson $^{6}$, P. Steier ${ }^{5}$, O. Tengblad ${ }^{3}$, and V. Vedia ${ }^{4}$ \\ 1 Department of Physics and Astronomy, Aarhus University, DK-8000, Aarhus C, Denmark \\ 2 ISOLDE, EP Department, CERN, CH-1211 Geneve 23, Switzerland \\ 3 Instituto de Estructura de la Materia, CSIC, E-28006 Madrid, Spain \\ 4 Grupo de Física Nuclear and IPARCOS, Universidad Complutense de Madrid, CEI Moncloa, E-28040 Madrid, Spain \\ ${ }^{5}$ Faculty of Physics, University of Vienna, Währinger Strasse 17, A-1090 Wien, Austria \\ 6 Institutionen för Fysik, Chalmers Tekniska Högskola, SE-41296 Göteborg, Sweden \\ 7 Helmholtz-Zentrum Dresden-Rossendorf, D-01328 Dresden, Germany
}

Received: date / Revised version: date

\begin{abstract}
We report on an attempt to reproduce the observation of $\beta^{-}$-delayed proton emission from ${ }^{11} \mathrm{Be}$ through detection of the final state nucleus ${ }^{10} \mathrm{Be}$ with accelerator mass spectrometry. Twelve samples were collected at the ISOLDE facility at CERN at different separator settings, allowing tests of different sources of contamination to be carried out. The observed amounts of ${ }^{10} \mathrm{Be}$ per collected ${ }^{11} \mathrm{Be}$ rule out several contamination sources, but do not agree internally. Formation of $\mathrm{BeH}$ molecular ions in the ion source may explain our data, in which case an upper limit of the $\beta$ p branching ratio of $2.2 \cdot 10^{-6}$ can be derived.
\end{abstract}

PACS. 23.40.-s $\beta$ decay $-27.20 .+\mathrm{n} 6 \leq \mathrm{A} \leq 19$

\section{Introduction}

Beta-decay has long been recognized as a powerful probe of nuclear structure. It gains further in versatility when moving to nuclei far from the line of beta-stability as betadelayed particle emission become energetically possible, see [1,2] for general reviews. Certain beta-delayed processes turn out 3 to occur only in near-dripline nuclei, the one we focus on here is beta-minus delayed proton emission. This process is only allowed for a few neutron-rich nuclei and was predicted 4 to have a very small branching ratio even for the most promising case, that of ${ }^{11} \mathrm{Be}$. The first experiments [5, 6, 7, to look for this decay focussed on detecting the final nucleus ${ }^{10} \mathrm{Be}$ rather than the emitted proton. In the latest of these [7] evidence for the decay was found with an intensity $8.3(9) \cdot 10^{-6}$, orders of magnitude above the preceeding theoretical prediction of $3.0 \cdot 10^{-8}$ [4] Apart from the intrinsic interest of this result, recent suggestions of alternative neutron decay branches [8, 9] have added motivation for the study of this particular decay. It is therefore important to have a careful assessment of possible systematic errors that may influence the obtained results. This paper describes a more extensive series of tests carried out to check the validity of the reported branching ratio.

An overview of the current state of knowledge on the beta decay of ${ }^{11} \mathrm{Be}$ was given in $[6$. A recent experimental

a email:kvr@phys.au.dk result [10] clarified the situation for the beta-delayed $\alpha$ decay branch of ${ }^{11} \mathrm{Be}$.

\section{Experimental procedures}

The basic idea is as in the earlier experiments [6,7] to collect ${ }^{11}$ Be samples, determine their intensity on-line via the $\gamma$-decays and measure the amount of produced ${ }^{10} \mathrm{Be}$ via a subsequent accelerator mass spectrometry (AMS) step. Samples collected at different settings of the ISOLDE mass separator allow checking the reproducibility of the result as well as testing for different sources of contamination. Figure 1 gives an overview of the experimental set-up at the source position.

The distance from the collection point to the outer side of the flange of the collection chamber is $31 \mathrm{~mm}$. A HPGe detector was positioned $632(2) \mathrm{mm}$ downstream from the flange in continuation of the beam line direction. $\mathrm{A} \mathrm{LaBr}_{3}(\mathrm{Ce})$ and a CEPA4 (combined $\mathrm{LaBr}_{3}(\mathrm{Ce}$ ) and $\mathrm{LaCl}_{3}(\mathrm{Ce})$ [1] ) detector were placed at either side of the chamber in the downstream direction in order not to block the line-of-sight to the HPGe detector, the distances from the flange of the chamber to the front edge of the $\mathrm{LaBr}_{3}$ and the CEPA4 were $88 \mathrm{~mm}$ and $126 \mathrm{~mm}$, respectively, corresponding to angles of 40-50 degrees from the beam line direction. The cylindrical $\mathrm{LaBr}_{3}$ detector had a radius of $10 \mathrm{~mm}$ and a length of $20 \mathrm{~mm}$, while the 




Fig. 1. The set-up: the ${ }^{11}$ Be beam is coming from the left, passes several collimators and is collected on the $\mathrm{Cu}$ sample that is mounted on the end flange. The on-line gamma activity is recorded in a HPGe at zero degrees (far right) and in LaBrdetectors positioned at both sides. See the text for details.

CEPA4 detector was formed by four squared crystals optically isolated, each of side length $27 \mathrm{~mm}$ and a lateral

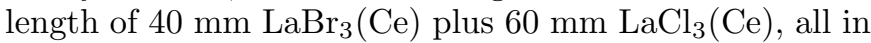
one encapsulation. Different DAQ systems were used for the HPGe (the standard ISOLDE MBS system) and for the La-detectors (a CAEN digitizer DTS730 coupled to a CAEN A2818). A pulser was added to the preamplifier of the HPGe to keep track of its deadtime; the deadtime in the digital chain is less than in the analogue chain.

In a two day collection campaign in May 2015 a total of twelve samples were collected at different separator settings in order to cross-check for possible systematic errors. After sample 5 was collected extra shielding, a $10.0 \mathrm{~mm}$ thick $\mathrm{Pb}$ plate and a $4.0 \mathrm{~mm}$ thick $\mathrm{Al}$ plate, was inserted at $10 \mathrm{~mm}$ distance from the HPGe detector to further reduce its count rate. The overall combined uncertainty in thickness is $0.5 \mathrm{~mm}$.

\subsection{Production and separation of ${ }^{11} \mathrm{Be}$}

The $1.4 \mathrm{GeV}$ proton beam from CERN's PS-Booster accelerator was directed onto a Ta target (95 g in foils of 20 $\mu \mathrm{m}$ and $6 \mu \mathrm{m}$ thickness placed in a $16 \mathrm{~g} \mathrm{~W}$ boat). The reaction products diffused out into a rhenium surface ion source where Be atoms were laser ionized employing the RILIS method 12. The laser settings were reoptimized on ${ }^{9} \mathrm{Be}$ at several occasions during the run. The ionization scheme depends on the mass of the isotope, but not strongly: a test on ${ }^{9} \mathrm{Be}$ with the ${ }^{10} \mathrm{Be}$ laser scheme gave an intensity reduction of about $35 \%$. A mass marker, containing stable $\mathrm{Na}$ and $\mathrm{Be}$, was used intermittently during the tuning of the separator and target, but was turned off two hours before the first collection started and was not used later on. The Be material in the mass marker was provided by Alfa Aesar as a solution in $5 \% \mathrm{HNO}_{3}$. The ions were accelerated through a $40 \mathrm{kV}$ gap, the spread of ion energy out of the ion source is typically a few eV.

The ion beam was mass separated in the High Resolution Separator (HRS). A set of slits positioned after the HRS magnets and before the RFQ cooler were adjusted to delimit the beam sent into the experimental set-up. The absolute positions of the slits were not available, but the beam full width at half maximum was estimated to be
Table 1. Mass values (in u) of selected isotopes and molecules, from [13, their mass difference to ${ }^{11} \mathrm{Be}$ and halflives.

\begin{tabular}{llll}
\hline & Mass $(\mathrm{u})$ & Mass difference $(\mathrm{u})$ & $\mathrm{T}_{1 / 2}$ \\
\hline${ }^{10} \mathrm{Be}$ & $10.01353470(9)$ & -1.0081263 & $1.39 \mathrm{My}$ \\
${ }^{10} \mathrm{Be}^{1} \mathrm{H}$ & $11.02135973(9)$ & -0.0003013 & $1.39 \mathrm{My}$ \\
${ }^{11} \mathrm{Be}$ & $11.02166108(26)$ & - & $13.8 \mathrm{~s}$ \\
${ }^{9} \mathrm{Li}^{1} \mathrm{H}_{2}$ & $11.04244025(20)$ & 0.0207792 & $178 \mathrm{~ms}$ \\
${ }^{11} \mathrm{Li}$ & $11.0437236(7)$ & 0.0220626 & $8.8 \mathrm{~ms}$ \\
\hline
\end{tabular}

about $1 \mathrm{~mm}$, and the final slit settings were $1.5 \mathrm{~mm}$ to the high-mass side and $6.5 \mathrm{~mm}$ to the low-mass side $(2.5 \mathrm{~mm}$ corresponds to a mass difference of $0.01 \mathrm{u}$ ). The masses of the isotopes and molecules that will be discussed later are given in table 1 . In the middle of the run (between samples 8 and 9) a scan of ${ }^{10}$ Be was carried out with a Faradaycup, the results are given in table 2 . They indicate that the effective width of the distribution for an isotope is less than $0.025 \mathrm{u}$. We note for later use that the intensity falls off by much more than two orders of magnitude when going $0.02 \mathrm{u}$ away from the nominal peak position. The shape is rather flat at the top with a quick fall off at the sides, which is consistent with a narrow peak with long tails passing a slightly wider slit. (This may be compared to the profile found for ${ }^{11} \mathrm{Be}$ in our previous experiment, figure 1 in 77.) Note that the scan was carried out using the ${ }^{11} \mathrm{Be}$ laser ionization scheme, demonstrating that ${ }^{10} \mathrm{Be}$ will be ionized with this setting.

The HRS allows the distributions of ${ }^{11} \mathrm{Li}$ and ${ }^{11} \mathrm{Be}$ to be separated, but tails of the neighbouring isobar may still be present. This is a potential source of contamination since ${ }^{10} \mathrm{Be}$ is produced in the largest decay branch of ${ }^{11} \mathrm{Li}$, beta-delayed one-neutron emission with a branching ratio about $85 \%$. To further suppress the amount of ${ }^{11} \mathrm{Li}$ in our sample we keep the beamgate closed for $150 \mathrm{~ms}$ after proton impact, only a tiny fraction of the $8.75 \mathrm{~ms}{ }^{11} \mathrm{Li}$ nuclei will survive this delay and will be collected.

The yield of ${ }^{11} \mathrm{Be}$ was at the beginning of the data taking measured in the ISOLDE tape station to $8 \cdot 10^{6}$ ions $/ \mu \mathrm{C}$ proton beam.

\subsection{The collection set-up and sample change}

As shown in figure 1 , the $40 \mathrm{keV}$ ion beam passed through three collimators (all three composed of a $1.5 \mathrm{~mm}$ thick $\mathrm{Cu}$ plate in front of an $\mathrm{Al}$ plate of thickness $20 \mathrm{~mm}, 20$ $\mathrm{mm}$ and $15 \mathrm{~mm}$, respectively, they were positioned 225 $\mathrm{mm}, 80 \mathrm{~mm}$ and $4 \mathrm{~mm}$ before the collection point and had circular opening of diameters $5 \mathrm{~mm}, 5 \mathrm{~mm}$ and 10 $\mathrm{mm})$ and was implanted in a small copper plate $(15 \times 20 \times 2$ $\mathrm{mm}$ ) mounted in a holder fixed to the end flange of the beamline. The flange was taken off for sample changing.

The twelve ${ }^{11} \mathrm{Be}$ samples and their subsequent chemical treatment is described below. After the final ${ }^{11}$ Be sample we collected a source of ${ }^{24} \mathrm{Na}$ that was used to extend the energy calibration of the gamma detectors up to 2.75 $\mathrm{MeV}$. 
Table 2. Mass scan of HRS using the ${ }^{10}$ Be current reading in a beamline Faraday cup (FC7480). Current readings below 1 pA are not reliable.

\begin{tabular}{lcccccccccc}
\hline mass setting $(\mathrm{u})$ & 9.994 & 10.004 & 10.009 & 10.014 & 10.019 & 10.024 & 10.029 & 10.034 & 10.039 & 10.089 \\
current $(\mathrm{pA})$ & 0.010 & 0.15 & 40 & 43 & 38 & 37 & 0.05 & 0.02 & 0.005 & $<0.001$ \\
\hline
\end{tabular}

An attempt was made to measure the current of the ion beam on the $\mathrm{Cu}$ plates, but no trustworthy readings could be obtained due to problems in applying a reliable suppression voltage.

\subsection{The gamma-ray detection}

The high-purity Ge-detector was energy and efficiency calibrated with standard sources of ${ }^{60} \mathrm{Co},{ }^{133} \mathrm{Ba}$ and ${ }^{152} \mathrm{Eu}$ placed at the collection point. The latter source had the best absolute intensity calibration of about $2 \%$, the gamma lines at $344.3 \mathrm{keV}, 1085.8 \mathrm{keV}, 1089.7 \mathrm{keV}, 1112.1 \mathrm{keV}$ and $1408.0 \mathrm{keV}$ were used for the efficiency calibrations, other lines were either too weak or too close to background lines. The ${ }^{60}$ Co source (lines at $1173.2 \mathrm{keV}$ and $1332.5 \mathrm{keV}$ ) and the ${ }^{24} \mathrm{Na}$ sample (lines at $1368.6 \mathrm{keV}$ and $2754.0 \mathrm{keV}$ ) could be used to extend the calibration up to $2750 \mathrm{keV}$. Calibrations were done separately for the two configurations, without and with $\mathrm{Pb}$ and $\mathrm{Al}$ plates inserted between the calibration source and detector, and gave final fullenergy peak efficiencies at $2124 \mathrm{keV}$ of $(5.4 \pm 0.2) \cdot 10^{-5}$ and $(2.7 \pm 0.2) \cdot 10^{-5}$, respectively. The calibration sources were also placed behind the collimators closest to the collection point; the detection efficiency in the range 1100-1400 keV went down by a factor six.

The $2124 \mathrm{keV}$ line is the dominating $\gamma$ line from ${ }^{11} \mathrm{Be}$. The one at $2896 \mathrm{keV}$ is also visible in our spectra, see e.g. figure 2, its intensity relative to the $2124 \mathrm{keV}$ line is the same for all samples where it appears and it gives a consistent yield estimate. Several other lines at higher energy were only observed in the $\mathrm{LaBr}$ detectors discussed below. The line at $478 \mathrm{keV}$ is discussed in section 2.3.2.

During the run we observed, apart from standard background gamma-lines such as from ${ }^{40} \mathrm{~K}$, gamma-lines from the decays of short-lived ${ }^{14} \mathrm{O},{ }^{24} \mathrm{Na},{ }^{10} \mathrm{C}$ and some weaker activities, most likely also ${ }^{7} \mathrm{Be}$ as discussed below. The intensity of the $15 \mathrm{~h}{ }^{24} \mathrm{Na}$ gamma-lines increased throughout the run, then decreased for three days and finally increased again when the following ISOLDE run started. These activities are therefore attributed to a background produced by proton impact on the ISOLDE targets, either to isotopes produced directly in the target region or produced by neutrons diffusing into the experimental hall. We checked that no such line interferes with the gamma-lines used in our analysis except for the $478 \mathrm{keV}$ line discussed below.

\subsubsection{Dead time}

In our previous experiment [7] the dead time was estimated via the ratio of accepted to total number of triggers. As an extra check, we here also estimate the dead

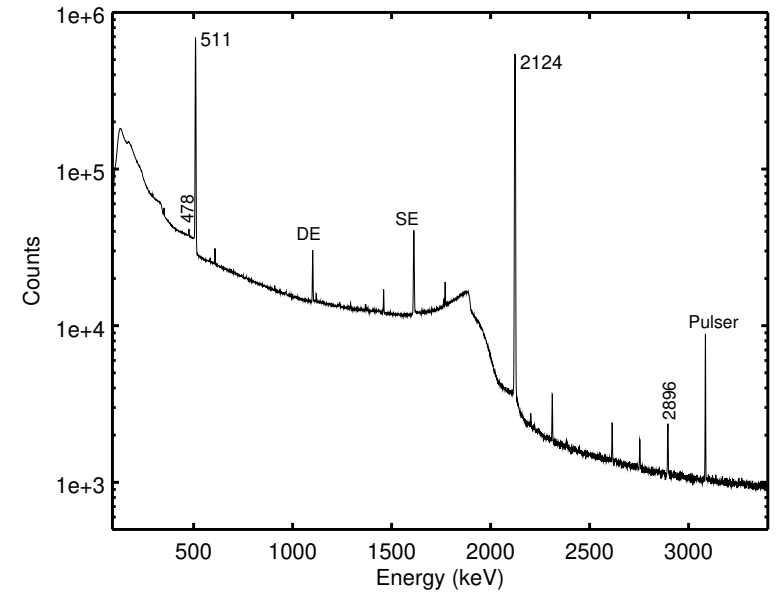

Fig. 2. The gamma-spectrum recorded from the ${ }^{11}$ Be beam in the HPGe detector from sample 6 . The major lines are marked by their energy in $\mathrm{keV}$. SE and DE denote single and double escape peaks from the $2124 \mathrm{keV}$ peak. Unmarked peaks are background peaks.

time by adding a pulser whose peak is positioned at an energy just above $3 \mathrm{MeV}$ in the spectrum from the HPGe detector.

The rate of the pulser was checked regularly during the run by comparison to an external clock. All the tests performed were consistent with a pulser rate of $1.001 \mathrm{~Hz}$ with a precision better than $5 \cdot 10^{-4}$. The background below the pulser peak in the gamma spectrum was noticeable for samples with a high ${ }^{11} \mathrm{Be}$ abundance and was estimated from the regions to both sides of the pulser peak. The deadtimes were deduced for each run by comparing the observed number of events in the pulser peak with the one expected from the duration of the run, the results are given in table 3 . The deadtimes were observed to be proportional within uncertainties to the count rate in the gamma spectrum, the proportionality extended over the interval from 0.1 to $5.5 \mathrm{kHz}$. The deadtime correction should be realiable to within one percent.

\subsubsection{The $478 \mathrm{keV}$ line}

The $478 \mathrm{keV}$ line originates in beta-delayed alpha-emission to the first excited state in ${ }^{7} \mathrm{Li}$. Reliable measurements of its branching ratio have only become available recently, the latest measurement gives $0.261(13) \%$ 6, 10. The line is emitted from the moving ${ }^{7} \mathrm{Li}$ nucleus while it is slowed down inside the sample. The recoil broadening gives rise to an almost triangular shape of the line. This is clearly visible in several spectra, see figure 3. In samples with 


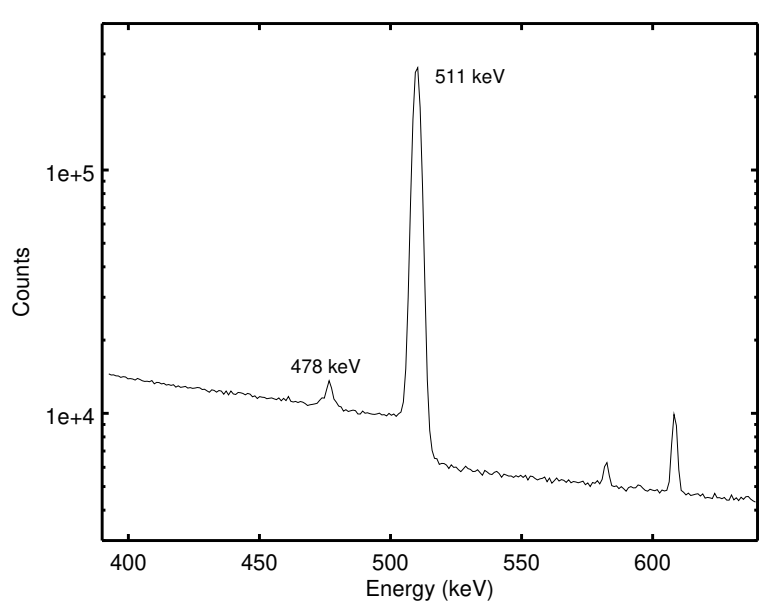

Fig. 3. Part of the gamma-spectrum recorded from the ${ }^{11} \mathrm{Be}$ beam in the HPGe detector from sample 2. The line at $478 \mathrm{keV}$ has contributions from a background source as well as from the sample, see text for details. Unmarked peaks are background peaks.

a small amount of ${ }^{11} \mathrm{Be}$ one still observes the $478 \mathrm{keV}$ line, but now with a narrow line shape (indicative of a non-moving source). This component is also present in the calibration measurements and is most likely due to a ${ }^{7}$ Be background source. Its intensity was determined to be $0.44(4) \mathrm{s}^{-1}$. In some samples, such as the one shown in figure 3 , both components are seen.

We have used the intensity of the broad component in order to estimate whether significant parts of the ${ }^{11} \mathrm{Be}$ activity could be placed outside of the $\mathrm{Cu}$ collection plate. It is much easier absorbed than the lines above $2 \mathrm{MeV}$, the absolute efficiency without the extra absorbers was determined as $7.2(1) \cdot 10^{-5}$ and the intensity in samples $\mathrm{S} 1$, S2 and S5 agreed with the one determined from the 2124 $\mathrm{keV}$ line. The extra $\mathrm{Pb}$ and $\mathrm{Al}$ absorbers should reduce its intensity further by a factor $0.15(2)$, again in fair agreement with the data. It is therefore highly unlikely that a substantial part of the ${ }^{11} \mathrm{Be}$ activity is situated outside of the $\mathrm{Cu}$ plate, but still visible for the HPGe detector. Such stray activity would suffer more absorption, which would give an imbalance between the $478 \mathrm{keV}$ and 2124 $\mathrm{keV}$ intensities.

\subsubsection{The La-detectors}

The $\mathrm{LaBr}_{3}(\mathrm{Ce})$ and CEPA4 detectors allow us to perform further checks. The La-detectors have much smaller volume than the HPGe detector so that absolute efficiencies are low, they are therefore placed closer to the source collection point. The La-detectors have as internal contaminants small activites of ${ }^{138} \mathrm{La}$ and ${ }^{227} \mathrm{Ac}$. The former gives gamma rays of energy $788.7 \mathrm{keV}$ and $1435.8 \mathrm{keV}$, the latter alpha particles that give detector signals in the range roughly from 2 to $2.5 \mathrm{MeV}$. These backgrounds are clearly visible in background spectra, but cannot be seen when high ${ }^{11} \mathrm{Be}$ activities are present.

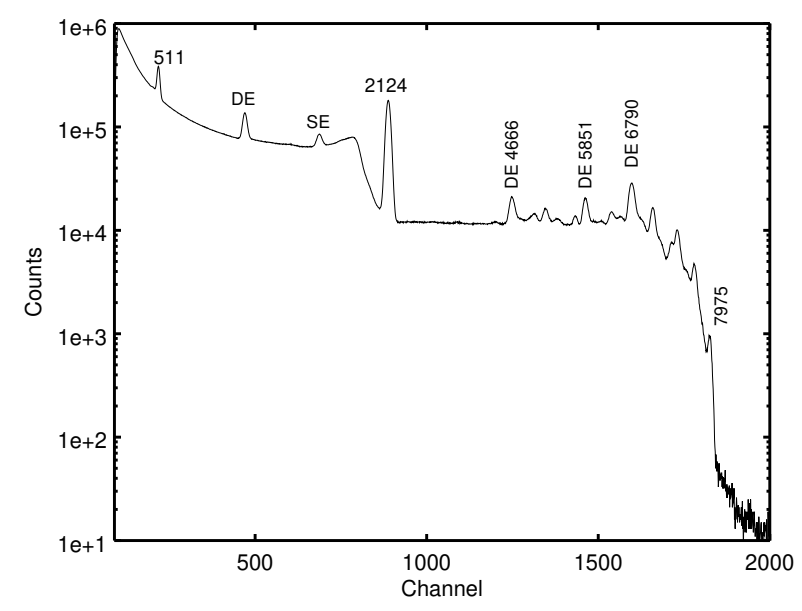

Fig. 4. The gamma-spectrum recorded from the ${ }^{11} \mathrm{Be}$ beam in the $\mathrm{LaBr}_{3}$ detector from sample 6. Note that the energy calibration is non-linear above $2 \mathrm{MeV}$. The major lines are denoted by their energy in $\mathrm{keV}, \mathrm{SE}$ and DE denote single and double escape peaks.

The intrinsic efficiency of the La-detectors is good at higher gamma energy so that the ${ }^{11} \mathrm{Be}$ gamma lines at $4665.9 \mathrm{keV}, 5851.5 \mathrm{keV}, 6789.8 \mathrm{keV}$ and $7974.7 \mathrm{keV}$ and their escape lines are clearly seen in the spectra. As an example the spectrum from sample 6 is shown in figure 4. The energy-channel relation is strongly non-linear above $2 \mathrm{MeV}$, indicating that the amplification of the photomultiplier tubes was set too high. Due to the small volume of the $\mathrm{LaBr}_{3}$ crystal the double escape peaks are more intense than the single escape peaks, which again are more intense than the full energy peaks.

The energy resolution is worse than for the HPGe detector and the lines therefore more sensitive to background components and other contamination lines. Nevertheless, they give a valuable check of the source strength of the samples as well as of the geometrical position of the ${ }^{11} \mathrm{Be}$ intensity. The absolute detection efficiencies at $2124 \mathrm{keV}$ are in the range of a few times $10^{-5}$ and the deduced source intensities are consistent with the ones from the HPGe analysis. A further test is possible using the highenergy part of the gamma spectrum, i.e. including all events above $3.5 \mathrm{MeV}$ whether they are in the full-energy peaks, in the escape peaks or in the Compton edge. The background is negligble here and the number of events about double the content of the $2124 \mathrm{keV}$ full-energy peak. The variation in relative count rates in the high-energy part and the $2124 \mathrm{keV}$ peaks (in the HPGe detector or the $\mathrm{LaBr}_{3}$ detector) across the samples is less than $10 \%$ for the $\mathrm{LaBr}_{3}$ detector and less than $20 \%$ for the CEPA4. This again indicates that all the visible ${ }^{11} \mathrm{Be}$ intensity is situated at the source collection point.

\subsection{Accelerator mass spectrometry}

The high sensitivity of accelerator mass spectrometry (AMS) was used to deduce the number of ${ }^{10} \mathrm{Be}$ atoms produced 
by beta-delayed proton emission from the collected ${ }^{11} \mathrm{Be}$. To perform the AMS measurements the ${ }^{10} \mathrm{Be}$ had to be first chemically enriched from the samples. To achieve this, the $\mathrm{Cu}$ plates were transferred to the Helmholtz-Zentrum Dresden-Rossendorf (HZDR), where beryllium was chemically extracted from the plates.

The first step was to partially dissolve the $\mathrm{Cu}$ sample in dilute nitric acid. As the ${ }^{11} \mathrm{Be}$ atoms impinging onto the target had an energy of only up to $40 \mathrm{keV}$ the implantation depth was below $1 \mu \mathrm{m}$. Because of that it was not necessary to bring the whole copper plate into solution. Instead only the surface was dissolved, which should include all ${ }^{10} \mathrm{Be}$ atoms. To check for possible missed ${ }^{10} \mathrm{Be}$ atoms and to exclude a possible intrinsic ${ }^{10} \mathrm{Be}$ contamination of the copper material a second successive partial dissolution step was performed for each $\mathrm{Cu}$ plate. The total weight of each copper plate was about $5 \mathrm{~g}$ and the amount of copper dissolved was ranging between 10 and $30 \mathrm{mg}$ for the first dissolution step and 14-36 mg for the second dissolution step. As AMS measures isotopic ratios and not absolute concentrations and to allow chemical separation, a well-defined amount of stable ${ }^{9} \mathrm{Be}$ was added to each solution. In this step it is very important to minimize the amount of ${ }^{10} \mathrm{Be}$ to be introduced together with the ${ }^{9} \mathrm{Be}$ material. Commercially available ${ }^{9}$ Be material usually has too high levels of ${ }^{10} \mathrm{Be}$ 14. About $300 \mu \mathrm{g}{ }^{9} \mathrm{Be}$ (as a $\mathrm{BeCl}_{2}$ solution) extracted from a dedicated low-level phenakite material was added to each sample. An aqueous solution of ammonia was added to the solution, which precipitates beryllium in the form of $\mathrm{Be}(\mathrm{OH})_{2}$. Copper forms ammonia complexes, which stay in the solution. After centrifugation and repeated rinsing, beryllium hydroxide was transferred into quartz crucibles where it was first dried on a hot plate and later ignited to beryllium oxide $(\mathrm{BeO})$ at $900^{\circ} \mathrm{C}$. The resulting amount of $\mathrm{BeO}$ from each sample was around 0.5 to $1.0 \mathrm{mg}$. The $\mathrm{BeO}$ was mixed with $\mathrm{Nb}$ powder and pressed into AMS sample holders. Beryllium oxide samples from twelve copper plates were produced in this way. In addition, a copper plate that was not exposed to any beam was treated in the same way to get a processing blank. This allows to check for the amount of ${ }^{10} \mathrm{Be}$ introduced in the chemical procedure. In total 26 AMS samples, i.e. 2 subsequent partial dissolutions of twelve irradiated and one blank $\mathrm{Cu}$ plate, were produced.

The AMS samples were transferred to the Vienna Environmental Research Accelerator (VERA) where the actual AMS measurement was performed in May 2016. VERA is a dedicated AMS facility based on a $3 \mathrm{MV}$ pelletron tandem accelerator from National Electrostatics Corporation (NEC) [15].

The prepared samples were introduced in a cesium sputter ion source and a beam of $\mathrm{BeO}^{-}$was extracted. After the tandem accelerator ions with charge state $2^{+}$ were selected. To separate the ${ }^{10} \mathrm{Be}$ ions from the ${ }^{10} \mathrm{~B}$ background a split-anode gas ionization chamber with a silicon nitride foil stack in front was used [16. Due to the higher nuclear charge of $\mathrm{B}$ compared to $\mathrm{Be}$ and the resulting higher stopping power the foil stack acts as a passive absorber reducing the amount of ${ }^{10} \mathrm{~B}$. The remaining boron ions entering the detector can be identified by their different energy loss in the two regions of the detector. The total efficiency of the AMS measurement is about $5 \cdot 10^{-4}$. To optimize and normalize the measurement pure BeO extracted from phenakite (machine blank) together with a standard sample, SMD-Be-12 [17, with a well-known ratio for ${ }^{10} \mathrm{Be} /{ }^{9} \mathrm{Be}$ of $1.704(30) \cdot 10^{-12}$ were measured together with our samples.

\section{Data analysis}

The final deduced number of ${ }^{11}$ Be decays in each sample is shown in table 3. The table also lists the collection time (uncertainty about $5 \mathrm{~s}$ ) and HPGe detector deadtime for each file, and the setting of the HRS mass separator. Most runs were performed with the beamgate being closed for the first $150 \mathrm{~ms}$ after proton impact on target and with the RILIS ionization scheme set for ${ }^{11} \mathrm{Be}$. The yield fluctuated during the whole run reaching a maximum of around $10^{7}$ ${ }^{11} \mathrm{Be} / \mathrm{s}$. The fluctuations were partly due to slow drifts in RILIS so the lasers were monitored and reoptimized when needed. In particular, at the end of collection of sample S10 the RILIS power was found to be very low so that no conclusions can be drawn from that sample. Sample S3 was on purpose taken with RILIS being off and sample S8 (on the mass of ${ }^{11} \mathrm{Li}$ ) was also taken with RILIS off and with an "inverted" beamgate, i.e. only open in the first $150 \mathrm{~ms}$ after proton impact.

The ${ }^{9} \mathrm{Li}^{1} \mathrm{H}_{2}^{+}$ion is stable [18] and may, if produced in our target and ion source, contribute to the beta count rate close to the ${ }^{11} \mathrm{Li}$ mass position. However, it is unlikely to be a dominating component and it will in any case not contribute to the production of ${ }^{10} \mathrm{Be}$.

The AMS analysis results are given in the last two columns of table 3. The amount of ${ }^{10} \mathrm{Be}$ found in the second dissolutions are in the range of $(1-1.5) \cdot 10^{4}$ atoms. In the first dissolution only the processing blank sample reaches this low value, whereas values around $1.9 \cdot 10^{4}$ atoms are found for samples S9, S10 and S11. The maximum amount of ${ }^{10} \mathrm{Be}$ found in the first dissolutions is two orders of magnitude higher.

Note that the number of ${ }^{10} \mathrm{Be}$ atoms observed for sample S9 converts into an upper limit of about 6 atoms/s for the ${ }^{10} \mathrm{Be}$ intensity at the mass setting $10.089 \mathrm{u}$. This is orders of magnitude below the upper limit on the current recorded for the same mass setting in table 2 where the maximum intensity, at the peak position, converts to $2.7 \cdot 10^{8}$ ions/s. This strongly indicates that the "high mass tail" of the ${ }^{10}$ Be distribution, which corresponds to ions that have higher kinetic energy than the average or have been scattered on the path through the magnets, will have negligible intensity at mass 11 . We note that this high level of purity would be difficult to obtain with a one stage magnetic separator. 
Table 3. For each sample, the separator setting, collection time, deadtime, the number of ${ }^{11}$ Be decays deduced from the HPGe spectra and the number of ${ }^{10} \mathrm{Be}$ atoms deduced for the first and second dissolutions are listed.

\begin{tabular}{llrcccc}
\hline Sample & Mass setting $(\mathrm{u})$ & Time $(\mathrm{s})$ & Deadtime $(\%)$ & Number of ${ }^{11}$ Be & First dissolution & Second dissolution \\
\hline S1 & 11.022 & 23323 & $20.5(3)$ & $2.41(15) \cdot 10^{11}$ & $(180 \pm 6) \cdot 10^{4}$ & $(1.51 \pm 0.33) \cdot 10^{4}$ \\
S2 & 11.037 & 15759 & $7.77(37)$ & $2.55(17) \cdot 10^{10}$ & $(8.0 \pm 0.8) \cdot 10^{4}$ & $(0.74 \pm 0.28) \cdot 10^{4}$ \\
S3 & $11.022^{a}$ & 8092 & $4.79(38)$ & $<2.7 \cdot 10^{6}$ & $(4.1 \pm 0.5) \cdot 10^{4}$ & $(1.22 \pm 0.31) \cdot 10^{4}$ \\
S4 & 11.012 & 7646 & $4.61(31)$ & $1.03(7) \cdot 10^{9}$ & $(6.3 \pm 0.7) \cdot 10^{4}$ & $(1.15 \pm 0.27) \cdot 10^{4}$ \\
S5 & 11.022 & 14590 & $13.6(6)$ & $7.4(5) \cdot 10^{10}$ & $(31.0 \pm 1.9) \cdot 10^{4}$ & $(1.08 \pm 0.32) \cdot 10^{4}$ \\
S6 & 11.022 & 24048 & $13.7(5)$ & $2.98(27) \cdot 10^{11}$ & $(67.0 \pm 3.4) \cdot 10^{4}$ & $(1.67 \pm 0.34) \cdot 10^{4}$ \\
S7 & 11.044 & 7846 & $6.44(33)$ & $2.2(4) \cdot 10^{7}$ & $(3.0 \pm 0.5) \cdot 10^{4}$ & $(0.80 \pm 0.32) \cdot 10^{4}$ \\
S8 & $11.044^{b}$ & 7676 & $6.00(33)$ & $<7.8 \cdot 10^{6}$ & $(126 \pm 5) \cdot 10^{4}$ & $(1.37 \pm 0.30) \cdot 10^{4}$ \\
S9 & 10.089 & 4290 & $6.12(42)$ & $<3.3 \cdot 10^{6}$ & $(1.91 \pm 0.35) \cdot 10^{4}$ & $(1.27 \pm 0.39) \cdot 10^{4}$ \\
S10 & $10.139^{c}$ & 8553 & $5.63(30)$ & $<5.5 \cdot 10^{6}$ & $(1.87 \pm 0.34) \cdot 10^{4}$ & $(1.01 \pm 0.27) \cdot 10^{4}$ \\
S11 & 11.007 & 11110 & $6.17(27)$ & $4.7(6) \cdot 10^{7}$ & $(1.97 \pm 0.35) \cdot 10^{4}$ & $(1.04 \pm 0.29) \cdot 10^{4}$ \\
S12 $^{c}$ & 11.030 & 10194 & $9.97(57)$ & $4.8(4) \cdot 10^{10}$ & $(8.5 \pm 0.8) \cdot 10^{4}$ & $(1.17 \pm 0.31) \cdot 10^{4}$ \\
blank $^{d}$ & & & & & $(1.08 \pm 0.31) \cdot 10^{4}$ & $(1.44 \pm 0.41) \cdot 10^{4}$ \\
BeO $^{e}$ & & & & & $(25.6 \pm 1.2) \cdot 10^{4}$ & \\
\hline
\end{tabular}

${ }^{a}$ RILIS blocked; ${ }^{b}{ }^{11} \mathrm{Li}$ run; ${ }^{c}$ low power in RILIS; ${ }^{d}$ processing blank, low ${ }^{10}$ Be-level phenakite; ${ }^{e}$ commercial BeO

\section{Discussion of results}

The observed line profile for ${ }^{11} \mathrm{Be}$ as the separator mass setting is increased is similar to the one seen in table 2 for ${ }^{10} \mathrm{Be}$, there is a steeper fall-off towards low mass settings (samples S11 and S4, in contrast to samples S12 and S2) consistent with the asymmetry of the slit settings. The different samples allow a systematic check of the three potentially worrying backgrounds that were also identified in our earlier work [6, 7.

The first is direct production of ${ }^{10} \mathrm{Be}$ being scattered to the wrong mass value somewhere in the separator. Our two steps in the magnetic separation and the electrostatic transport makes such a background rather unlikely, but the ${ }^{10} \mathrm{Be}$ peak is more intense than ${ }^{11} \mathrm{Be}$. It is therefore crucial that samples S9 and S11 have very little ${ }^{10} \mathrm{Be}$ content. As argued in the previous section this rules out this background source.

The second possibility is direct production of ${ }^{11} \mathrm{Li}$ whose largest decay branch produces ${ }^{10} \mathrm{Be}$. We do indeed see clear ${ }^{10} \mathrm{Be}$ production in sample $\mathrm{S} 8$ where we explicitly run for collecting ${ }^{11} \mathrm{Li}$. The intensity corresponds to collecting $190(8)^{11} \mathrm{Li} / \mathrm{s}$, which is a reasonable yield at these running conditions for the target. The corresponding gamma lines from the ${ }^{11} \mathrm{Li}$ decay were too weak to be seen, as would be expected. The crucial point is that for sample S7 at our standard running conditions very little of the ${ }^{11} \mathrm{Li}$ leaked through, at most $3 \%$. This amount is further reduced by more than two orders of magnitude when moving $0.02 \mathrm{u}$ down (as noted above), so we can safely conclude that the possible contamination from ${ }^{11} \mathrm{Li}$ will be much less than the signal observed on the ${ }^{11}$ Be mass.

The third possibility is ionization without break-up of neutral ${ }^{10} \mathrm{Be}^{1} \mathrm{H}$ molecules, a signal that would appear directly on the ${ }^{11} \mathrm{Be}$ position. We have earlier [6, 7] argued based on the known properties of this molecule [19] that it is highly unlikely that laser ionization gives a molecular ion rather than breaking up the molecule, in particular in
Table 4. Branching ratios for beta-delayed proton decay of ${ }^{11}$ Be derived from the values in table 3

\begin{tabular}{lccc}
\hline Sample, mass centred & $\mathrm{S} 1$ & $\mathrm{~S} 5$ & $\mathrm{~S} 6$ \\
Branch $\left(10^{-6}\right)$ & $7.4(5)$ & $3.9(4)$ & $2.2(2)$ \\
\hline Sample, off centre & $\mathrm{S} 12$ & $\mathrm{~S} 2$ & $\mathrm{~S} 4$ \\
Branch $\left(10^{-6}\right)$ & $1.4(2)$ & $2.4(4)$ & $43(8)$ \\
\hline
\end{tabular}

the strong laser fields in the source. If this is correct molecular ions are surface ionized and their yield should be at least the same when the lasers are turned off, i.e. the conditions for sample S3. We see very little signal there, which excludes surface ionization of neutral $\mathrm{BeH}$ molecules in the ion source as significant background to the signal at mass $11.022 \mathrm{u}$. If the small BeH signal from sample S3 is indicative of a non-zero contribution it would be peaked at the ${ }^{10} \mathrm{Be}^{1} \mathrm{H}$ mass and should therefore at other mass values decrease in intensity in a similar way that the ${ }^{11} \mathrm{Be}$ intensity does.

Turning now to the deduced branching ratios for betadelayed proton emission, these are collected in table 4 and divided into samples taken on the central mass value and those taken sligthly off mass, the latter ordered after the observed number of ${ }^{11} \mathrm{Be}$. To evaluate the branching ratios a background of $1.9 \cdot 10^{4}$ atoms was subtracted from the number of ${ }^{10} \mathrm{Be}$ atoms. (The sensitivity limit of our set-up would therefore, for collections of duration up to a day, correspond to branching ratios of order $10^{-7}$.) The result for sample S4 is surprisingly high, but we note that it corresponds to the lowest recorded numbers of ${ }^{10} \mathrm{Be}$ and ${ }^{11} \mathrm{Be}$ out of the six samples shown. For the other five samples, the overall order of magnitude is similar to that obtained earlier, namely the value of $2.5(2.5) \cdot 10^{-6}$ from [6] and the value of $8.3(9) \cdot 10^{-6}$ from [7], but the new values do clearly not agree internally. 


\subsection{Conjecture}

One possible interpretation of the disagreeing branching ratios could be the presence of a source of ${ }^{10} \mathrm{Be}$ that is displaced slightly below the ${ }^{11} \mathrm{Be}$ mass values and decreases in intensity with time. A gradual ${ }^{10} \mathrm{Be}$ intensity decrease could explain the decreasing branching ratios for the samples on the central mass value: S1, S5 and S6. The displacement of the source would be needed to explain why $\mathrm{S} 2$ is low (and similar for S12) and, in particular, why S4 is much higher than the other values: From sample S4 to sample S5 the ${ }^{11} \mathrm{Be}$ intensity per time increases a factor $38(3)$, whereas the increase for ${ }^{10} \mathrm{Be}$ is an order of magnitude less, a factor 3.5(6). From sample S1 to sample S2 the corresponding intensities per time decrease by factors of $6.4(6)$ and $20(3)$ for ${ }^{11} \mathrm{Be}$ and ${ }^{10} \mathrm{Be}$ (in this case part of the change could be due to a drop in the target yield since the deadtime was observed to decrease slightly during collection of sample S1). This pattern suggests that the ${ }^{10} \mathrm{Be}$ intensity peaks at lower mass values than the ${ }^{11}$ Be intensity.

A simple estimate can be made by assuming Gaussian intensity profiles, using the mass difference of $0.0003 \mathrm{u}$ between ${ }^{10} \mathrm{Be}^{1} \mathrm{H}$ and ${ }^{11} \mathrm{Be}$ from table 1 and a width parameter of $0.0017 \mathrm{u}$ derived from the beam profile estimate in section 2.1. This gives the correct trend, but with too small intensity differences for the two components. The beam profile will be more complex than a Gaussian, but this nevertheless may point towards a larger effective mass difference than $0.0003 \mathrm{u}$.

The following hypothetical mechanism might explain our observations. We stress that we do not have any direct evidence for it, but present it as the only coherent - and possibly plausible - explanation we have been able to find for our data.

The suggestion is that ${ }^{10} \mathrm{Be}^{1} \mathrm{H}^{+}$is formed from ionized ${ }^{10} \mathrm{Be}^{+}$reacting with hydrogen (or water vapour) during the brief interval during which the ion is extracted from the ion source. ISOLDE targets contain hydrogen in small amounts and it is pumped out rather slowly when targets are put under vacuum. The gradual reduction of the amount of available hydrogen could then explain the decreasing size of our signal. The modelling of the chemical reaction inside (or on the way out of) the ion source will be complex. Our laser setting did ionize ${ }^{10} \mathrm{Be}$ so we are looking at processes with relative probability of order $10^{-7}$. However, some supporting evidence comes from the following observations of molecular beams in related environments:

At ISOLTRAP an experiment on ${ }^{79} \mathrm{Cu}^{+}$gave evidence for the molecule ${ }^{65} \mathrm{Cu}^{12} \mathrm{CH}_{2}^{+}$in a MR-TOF spectrum [20]. A resonance ionization mass spectrometry experiment at the University of Mainz identified a background of ${ }^{40} \mathrm{CaH}^{+}$ ions produced by collisions of ${ }^{40} \mathrm{Ca}^{+}$with hydrogenous residual gas molecules 21, the experimental conditions giving a relative intensity of $2 \cdot 10^{-5}$ for the hydride. At LISOL 22] (and other places) detailed studies of the interaction of ions with buffer gas- and impurity molecules led to observations of e.g. $\mathrm{Co}\left(\mathrm{H}_{2} \mathrm{O}\right)^{+}$.
The suggested mechanism would also be expected to be present at the earlier beamtimes. The large signal reported in 7] was from a sample taken shortly after the target had been put on-line and may therefore be due to the same effect. The smaller signal reported in [6] could be due to different purity of the target, e.g. a sample taken at a later time. A similar explanation may apply for the limit of $2 \cdot 10^{-6}$ of ${ }^{11} \mathrm{Be}$ on mass ${ }^{12} \mathrm{Be}$ quoted in [7.

Two obvious future checks of this mechanism are to look for ${ }^{12} \mathrm{Be}$ on mass ${ }^{13} \mathrm{Be}$ and for ${ }^{7} \mathrm{Be}$ on mass ${ }^{8} \mathrm{Be}$. If the mechanism can be confirmed there are several ways to reduce its effect. One may run with a narrow-band laser setting for ${ }^{11} \mathrm{Be}$ to reduce the simultaneous ionization of ${ }^{10} \mathrm{Be}$, or one may attempt to dissociate the $\mathrm{BeH}$ molecules, one possibility being to pass the ion beam through a thin C-foil (or a gas target).

\section{Summary and outlook}

The aim of this experiment was to check our previous claim [7] of a surprisingly large branching ratio of 8.3(9) . $10^{-6}$ for beta-delayed proton decay of ${ }^{11} \mathrm{Be}$. The variation of separator settings for the different collected samples has allowed to exclude all previously considered sources of contamination. Still, the current results for the branching ratio are inconclusive and not consistent with the previous value. We conjecture that a better understanding of molucular formation in the ion source is needed, and that there is a clear risk that our signal stems from this mechanism.

At the current level of understanding the most likely interpretation of the branching ratio results collected in table 4 is that a background source dominates all values execpt possibly the one from sample S12. A conservative $95 \%$ confidence level upper limit of $2.2 \cdot 10^{-6}$ for the branching ratio for beta-delayed proton decay can be obtained from sample S12 from the ratio of the number of ${ }^{10} \mathrm{Be}$ and ${ }^{11} \mathrm{Be}$ atoms without any background subtraction. We note that if neutron dark decays are responsible for the neutron decay anomaly [8, a sizable part of the energy range for the new "dark" particles is excluded by our limit 9 .

We outlined above possible ways to improve upon the present procedure for measuring the decay, but of course encourage experiments with alternative procedures, preferably ones that detect the proton. Due to the low branching ratio such experiments are rather difficult, but the recent interest in this decay has prompted several groups to attempt detection inside a TPC either by optical detection at ISOLDE 23 or electrical detection at TRIUMF 24]. As the main background in such detectors could be the beta-delayed alpha branch such experiments should be sensitive down to an interesting region of branching ratios. The recent report from the TRIUMF experiment [24] of a positive identification of the beta-delayed proton decay with a branching ratio of $13(3) \cdot 10^{-6}$ and a narrow proton energy distribution peaking around $178(20)$ $\mathrm{keV}$ is very interesting. This value does not agree with our upper limit. However, one possible explanation may 
be that the TRIUMF experiment observed (at least partially) the beta-delayed triton branch, since protons and tritons would be more difficult to distinguish in their setup. Our results only address the $\mathrm{p}+{ }^{10} \mathrm{Be}$ channel.

We would like to thank the ISOLDE technical teams for their efforts and T. Andersen, J. Heinemeier, B. Marsh, H. Ravn, S. Rothe, P. Van Duppen and K. Wendt for discussions. We would like to acknowledge support from the European Union's Horizon 2020 research and innovation programme under grant agreement no. 654002, from the Spanish research projects FPA201564969-P, FPA2015-65035-P and FPA2017-87568-P (MINECO/FEDER, $\mathrm{UE}$ ), and from the Danish Council for Independent Research (DFF - 4181-00218).

\section{References}

1. M. Pfützner, M. Karny, L.V. Grigorenko and K. Riisager, Rev. Mod. Phys. 84, (2012) 567.

2. B. Blank and M.J.G. Borge, Prog. Part. Nucl. Phys. 60 , (2008) 403.

3. B. Jonson and K. Riisager, Nucl. Phys. A693, (2001) 77.

4. D. Baye and E.M. Tursonov, Phys. Lett. B 696, (2011) 464.

5. J.C. Angélique et al., IS374 experiment proposal, CERN/ISC 98-6 ISC/P99 (1998).

6. M.J.G. Borge, L.M. Fraile, H.O.U. Fynbo, B. Jonson, O.S. Kirsebom, T. Nilsson, G. Nyman, G. Possnert, K. Riisager and O. Tengblad, J. Phys. G 40, (2013) 035109.

7. K. Riisager, O. Forstner, M.J.G. Borge, J.A. Briz, M. Carmona-Gallardo, L.M. Fraile, H.O.U. Fynbo, T. Giles, A. Gottberg, A. Heinz, J.G. Johansen, B. Jonson, J. Kurcewicz, M.V. Lund, T. Nilsson, G. Nyman, E. Rapisarda, P. Steier, O. Tengblad, R. Thies and S.R. Winkler et al., Phys. Lett. B 732, (2014) 305.

8. B. Fornal and B. Grinstein, Phys. Rev. Lett. 120, (2018) 191801.

9. M. Pfützner and K. Riisager, Phys. Rev. C97, (2018) 042501.

10. J. Refsgaard, J. Büscher, A. Arokiaraj, H.O.U. Fynbo, R. Raabe, and K. Riisager, Phys. Rev. C99 (2019) 044316.

11. E. Nácher, M. Mårtensson, O. Tengblad, H. Álvarez-Pol, M. Bendel, D. Cortina-Gil, R. Gernhäuser, T. Le Bleis, A. Maj, T. Nilsson, A. Perea, B. Pietras, G. Ribeiro, J. Sánchez del Río, J. Sánchez Rosado, A. Heinz, B. Szpak, M. Winkel, M. Zieblinski, Nucl. Instr. Meth. A769, (2015) 105.

12. J. Lettry, R. Catherall, G. J. Focker, O. C. Jonsson, E. Kugler, H. Ravn, C. Tamburella, V. Fedoseyev, V. I. Mishin, G. Huber, V. Sebastian, M. Koizumi, and U. Köster, Rev. Sci. Instr. 69, (1998) 761.

13. Meng Wang, G. Audi, F.G. Kondev, W.J. Huang, S. Naimi, Xing Xu, Chinese Physics C41, (2017) 030003.

14. S. Merchel et al., Nucl. Instr. Meth. B266, (2008) 4921.

15. P. Steier et al., Nucl. Instr. Meth. B223, (2004) 67.

16. P. Steier et al., Int. J. Mass Spectrometry 444, (2019) 116175 .

17. S. Akhmadeliev, R. Heller, D. Hanf, G. Rugel, S. Merchel, Nucl. Instr. Meth. B294, (2013) 5.

18. C. Sanz, E. Bodo and F.A. Gianturco, Chemical Physics 314, (2005) 135.
19. S. Bubin and L. Adamowicz, J.Chem.Phys. 126, (2007) 214305.

20. A. Welker et al., Phys. Rev. Lett. 119, (2017) 192502.

21. P. Müller et al., Fresenius J. Anal. Chem. 370, (2001) 508.

22. Yu. Kudryavtsev et al., Nucl. Instr. Meth. B204, (2003) 336.

23. A.A. Ciemny et al., CERN-INTC-2016-048/INTC-P-479.

24. Y. Ayyad et al., Phys. Rev. Lett. 123, (2019) 082501. 